- Wearing a removable prosthesis during the transitional phase may deter some patients from pursuing dental implant therapy.

- Transitioning with a fixed prosthesis prevents transmucosal loading of the implants and allows grafting procedures to be carried out.

- Immediate loading of dental implants provides an opportunity to provide patients with a fixed prosthesis on the day of surgery.

\title{
Transitioning patients from teeth to implants
}

\author{
S. Jivraj ${ }^{1}$ and W. Chee $^{2}$
}

One major obstacle to treatment with implants is the transitional phase between a tooth supported occlusion and an implant supported occlusion. This is of particular concern when a patient with a failing dentition has not worn a removable prosthesis before and is planning to have a reconstruction supported by implants.

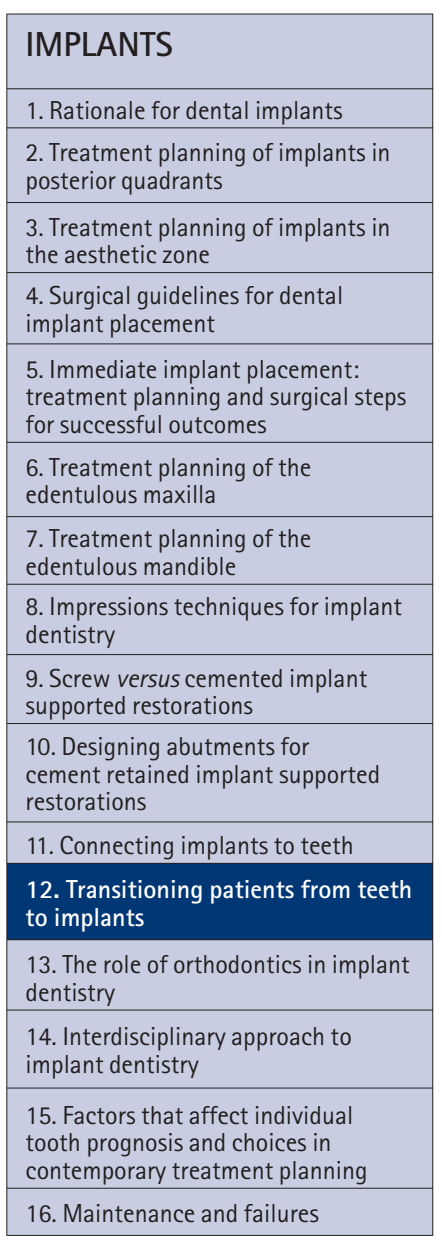

Implants were originally designed with the edentulous patients in mind, to compensate for the poor support and retention that was available from the mucosa. ${ }^{1,2}$

With conventional delayed loading protocols, patients receiving implants are sometimes required to wear a removable transitional prosthesis. For many patients this is unacceptable both socially and aesthetically.

Achieving osseointegration is not the challenge that it seemed to be 30 years ago. Today implant integration is a given and providing an implant supported restoration which is in harmony with the surrounding hard and soft tissues is the goal. Bone and gingival grafting procedures are routinely used to optimise the site prior to implant placement. Removable prostheses can hamper these procedures as pressure

\footnotetext{
${ }^{1 *}$ Chairman, Section of Fixed Prosthodontics and Operative Dentistry, University of Southern California School of Dentistry / Private Prosthodontics Practitioner, Burbank, California; ${ }^{2}$ Ralph W. and Jean L. Bleak Professor of Restorative Dentistry, Director of Implant Dentistry at the University of Southern California School of Dentistry / Private Prosthodontics Practitioner, Pasadena, California *Correspondence to: Dr Sajid Jivraj, 300 Esplanande Drive 1600, Oxnard CA 93036, USA Email: saj.jivraj@gmail.com
}

\section{Refereed Paper}

( ) British Dental Journal 2006; 201: 699-708 DOI: $10.1038 /$ sj.bdj.4814302 from these removable restorations can deter from proper healing of grafts.

The purpose of this article is to describe options other than removable prostheses in transitioning the patient from teeth to implant supported restorations. Emphasis is placed on fixed transitional prostheses for the reasons described above.

\section{Transitioning patients from teeth to implants} Today's patients have high expectations regarding aesthetics and providing functional restorations alone may not be sufficient to satisfy many of them. With heightened aesthetic expectations, it becomes imperative that the restorative dentist understand the patient's desires and expectations prior to embarking upon any irreversible therapy. Moreover, emphasis should be placed on diagnosis and treatment planning because in most situations the proper diagnosis will dictate the appropriate treatment plan. Inadequately planned treatment and inadequately informed patients, even when well executed, will result in less than ideal treatment satisfaction. ${ }^{3}$

The functional and aesthetic replacement of missing teeth with dental implants remains a complex task under most conditions. With comprehensive treatment planning and proper surgical and restorative protocols, more predictable results can be achieved. With this in mind, transitioning the patient from teeth to implant supported restoration becomes even more 
Fig. 1 Missing tooth 23 has been treatment planned for an implant restoration. The patient wanted to avoid a removable prosthesis in the transitional phase.

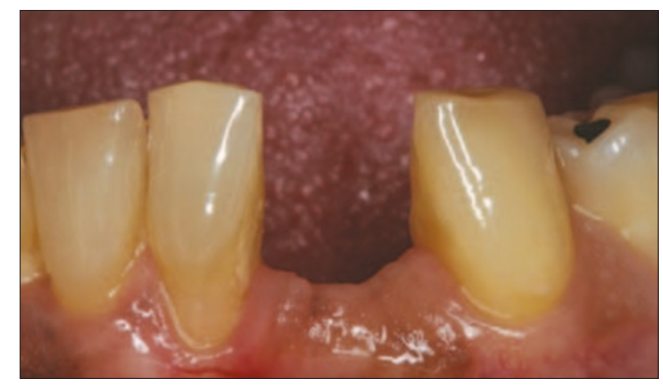

Fig. 2 A resin bonded fixed partial denture is a convenient way of transitioning the patient. It is entirely tooth supported and avoids putting pressure on the implant site.

Fig. 3 Facial view illustrating failing fixed partial denture from teeth 7-10. The patient wanted to avoid a removable prosthesis in the transitional phase.

Fig. 4 Occlusal view of failing fixed partial denture.

Fig. 5 Diagnostic wax up for 4 unit resin bonded fixed partial denture.

Fig. 6 Diagnostic wax up with cast metal framework from which a provisional restoration will be fabricated.
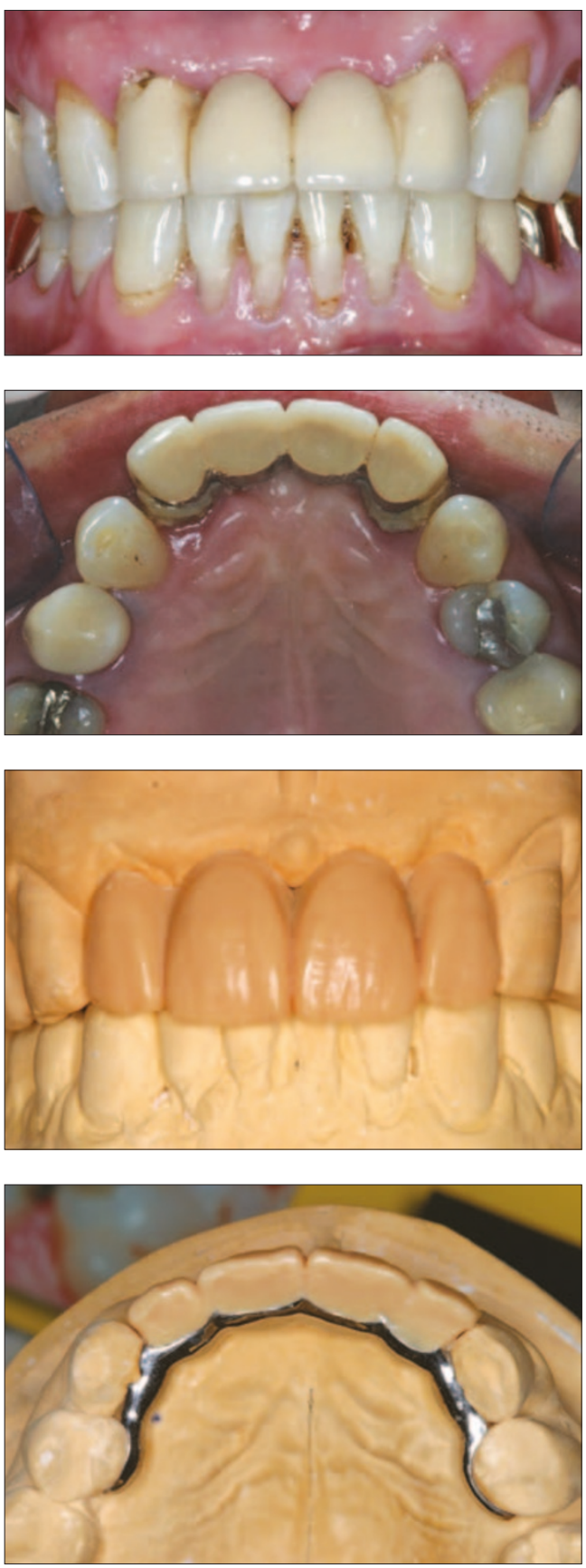

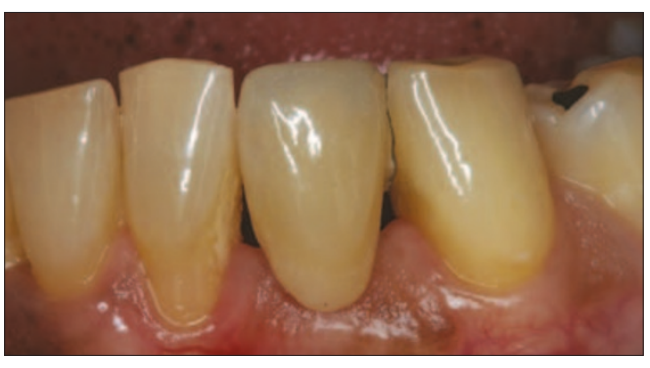

difficult as precise planning will include timing of extractions, method of retaining fixed provisional restorations, manipulation of remaining teeth to enhance implant receptor sites and many other issues. Patient preference is almost always to have a fixed prosthesis during this transition.

The rationale for fixed transitional prostheses suits both the patient and the clinician. Patient management with a fixed prosthesis has the advantages of elimination of the discomfort experienced with a removable prosthesis due to impingement of the mucosa. The physiological advantage of having a fixed restoration during integration of the implants is preventing transmucosal loading of the implants and not interfering with any grafting procedures. The clinician also benefits by decreased chair time, fewer unscheduled visits and reduced maintenance of the provisional prosthesis.

One disadvantage of using a fixed prosthesis in transition is the additional cost involved. However, in patients embarking upon already costly and lengthy therapy, the additional cost for comfort and convenience outweighs the social embarrassment of wearing a removable prosthesis.

There are several methods to transition a patient from teeth to implant supported restorations. Each has its own indication. The following methods will be discussed:

1. Resin bonded fixed partial dentures

2. Use of strategic abutments and provisional

fixed partial dentures

3. Provisional implants

4. Immediate loading.

\section{Resin bonded fixed partial dentures.}

Since the pioneering work of Rochette, ${ }^{5}$ there has been a plethora of techniques developed to enhance the success of resin bonded fixed partial dentures. ${ }^{6}$ The literature is quite clear in that resin bonded fixed partial dentures have achieved clinical success over time and there are indications for their use as definitive restorations. ${ }^{7}$ When treatment planning implant supported restorations, adhesively retained fixed partial dentures are a useful tool. They can be bonded to a single abutment tooth (Figs 12). The advantages of this are that the implant is not loaded, the patient has the convenience of a fixed prosthesis and no tooth structure is removed from the abutment tooth.

Transitioning patients when implants replace teeth in the aesthetic zone is the most difficult exercise. Orthodontic extrusion of teeth is an often-used tool to modify soft tissue levels. ${ }^{7}$ This has been discussed in part 3 of the series. When patients refuse the display of orthodontic brackets, resin bonded fixed partial dentures can serve to replace missing teeth while effecting the extrusion of teeth using magnets embedded to tooth roots and underside of pontics. Once the extrusion is complete the implants can be placed and the same prosthesis can be used to provide aesthetics during the period of 
integration. One disadvantage of this procedure is that it can be inconvenient since it requires replacement of the magnets at intervals and frequent de- and re-bonding. The patient must be made aware of the financial implications of such a prosthesis at the outset of treatment and the clinician must set his/her fees accordingly based on time spent completing the procedure.

The patient in Figure 3 presented with a failing 4 unit fixed partial denture spanning the maxillary incisors with the lateral incisors being the abutments. The patient decided that implant supported restorations would be more stable and have more options should failure occur. One prerequisite for treatment was that aesthetics be maintained throughout therapy and no removable prostheses would be tolerated. An analysis of the anterior aesthetics revealed that teeth 6 and 11 exhibited gingival recession and the gingival margins of the lateral incisors were more apical than the central incisors (Figs $3-4)$. When pointed out to the patient, it was requested that these deficiencies be addressed. A combined periodontal/orthodontic/prosthodontic approach was discussed with the patient which included grafting of the canines and orthodontic site development of the lateral incisors for placement of dental implants. The purpose of the orthodontics was to shift the periodontium of the lateral incisors more coronally to allow more pleasing proportions of the implant supported restoration. The patient continued to refuse any display of orthodontic hardware. As an alternative to conventional orthodontic hard ware, a provisional resin bonded fixed partial denture was fabricated (Figs 5-7). The maxillary lateral incisors were decoronated and the root face was prepared to recieve a magnet. The gingival surface of the provisional restoration was also prepared to receive a magnet. The magnet was attached using polymethyl methacrylate resin (Figs 8-13). The provisional fixed partial denture was bonded to adjacent canines and first premolars using a dual cured resin cement.

Although unconventional and somewhat inconvenient, the above procedure satisfied the patient's aesthetic demands of a fixed aesthetic transitional prosthesis while at the same time allowing the clinician to extrude the lateral incisors. Once the implants are placed the same prosthesis will be used as a provisional prosthesis during the integration phase. The added advantage of this procedure is that the implants are not loaded transmucosally (Figs 13-15).

\section{Use of strategic abutments}

Complex reconstructions that require implants for support take time to complete; this length of treatment is exacerbated when bone grafts are required. Maintaining some natural tooth abutments for this period of time to retain a fixed provisional restoration is one of the best methods to maintain function and aesthetics while the graft and implant sites are healing. The advantages of a fixed prosthesis are similar to those described earlier for the adhesive type

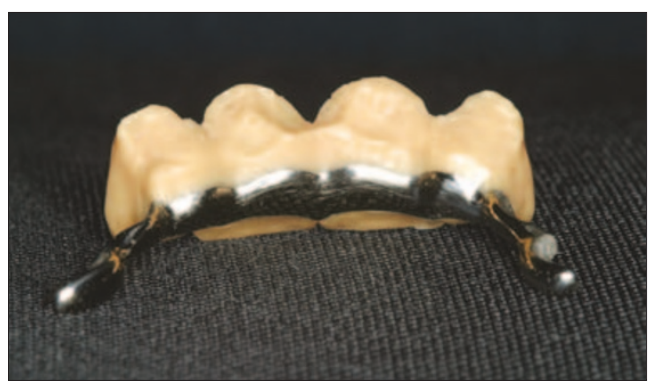

Fig. 7 Palatal view illustrating diagnostic wax up and framework design.

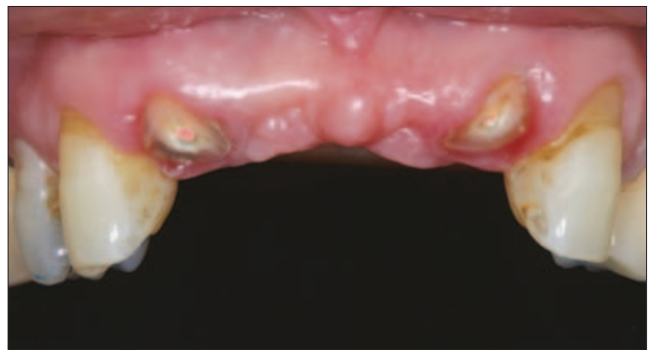

Fig. 8 Teeth 7 and 10 have been endodontically treated and decoronated to allow space for the transitional prosthesis.

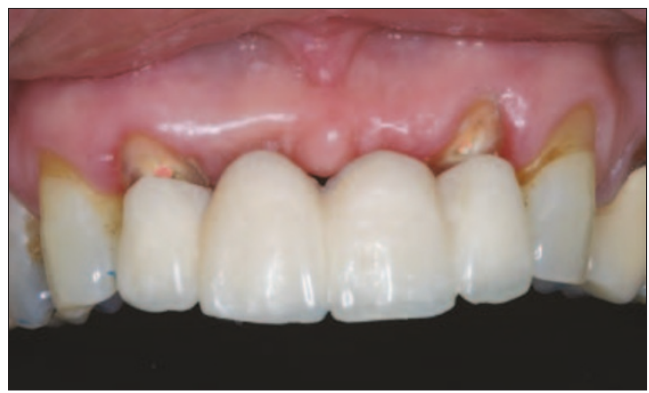

Fig. 9 Try in of transitional prosthesis.

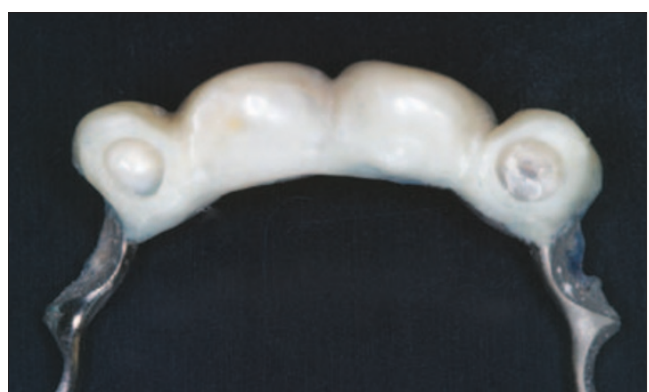

Fig. 10 Space made in gingival surface of provisional restoration to incorporate magnets.

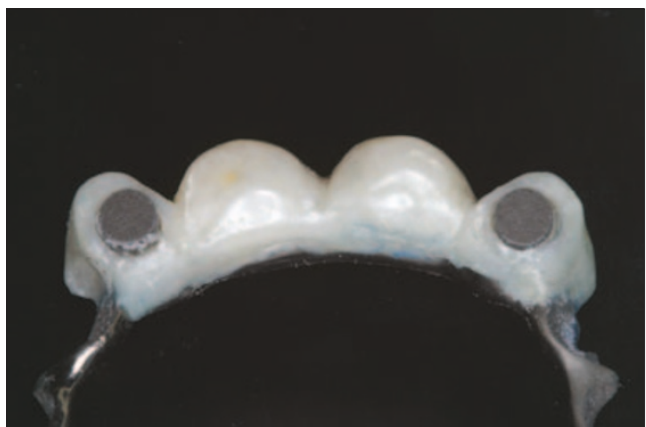

Fig. 11 Magnets incorporated into gingival surface of provisional restoration.

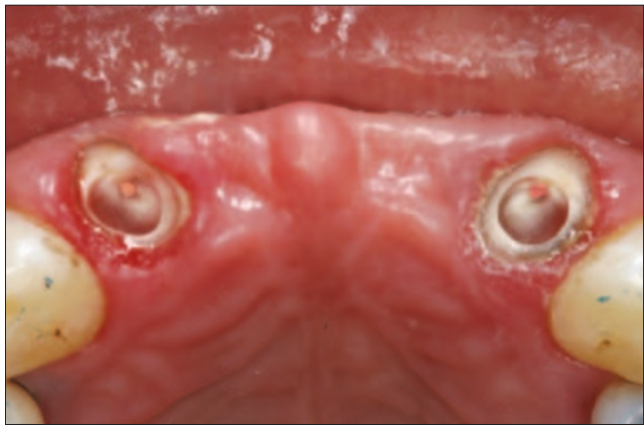

Fig. 12 Root face of abutments prepared to receive magnets. 
Fig. 13 (left) Magnets incorporated into root face using polymethyl methacrylate resin.

Fig. 14 (right) Facial view of transitional prosthesis in situ.
Fig. 15 (left) Smile view illustrating aesthetics of provisional restoration. The graying at the gingival margin is due to the magnet showing through.

Fig. 16 (right) Patient smile illustrating low lip line.

Fig. 17 (left) Facial view illustrating periodontally failing dentition, the patient has been treatment planned for full arch extractions and fabrication of an implant supported prosthesis. The patient wants to avoid a removable prosthesis in the transitional phase.

Fig. 18 (right) Canines and molars have been maintained on both sides to retain a provisional prosthesis.

Fig. 19 (left) Provisional restoration. This can also have gutta percha markers incorporated and function as a radiographic guide.

Fig. 20 (right) Extractions and delivery of provisional restoration.

Fig. 21 (left) Facial view of

provisional restorations on the day of extraction.

Fig. 22 (right) Facial view of provisional restorations following a week of healing.

Fig. 23 (left) Lateral view of patient with periodontally failing dentition.

Fig. 24 (right) Facial view of patient with periodontally failing dentition.
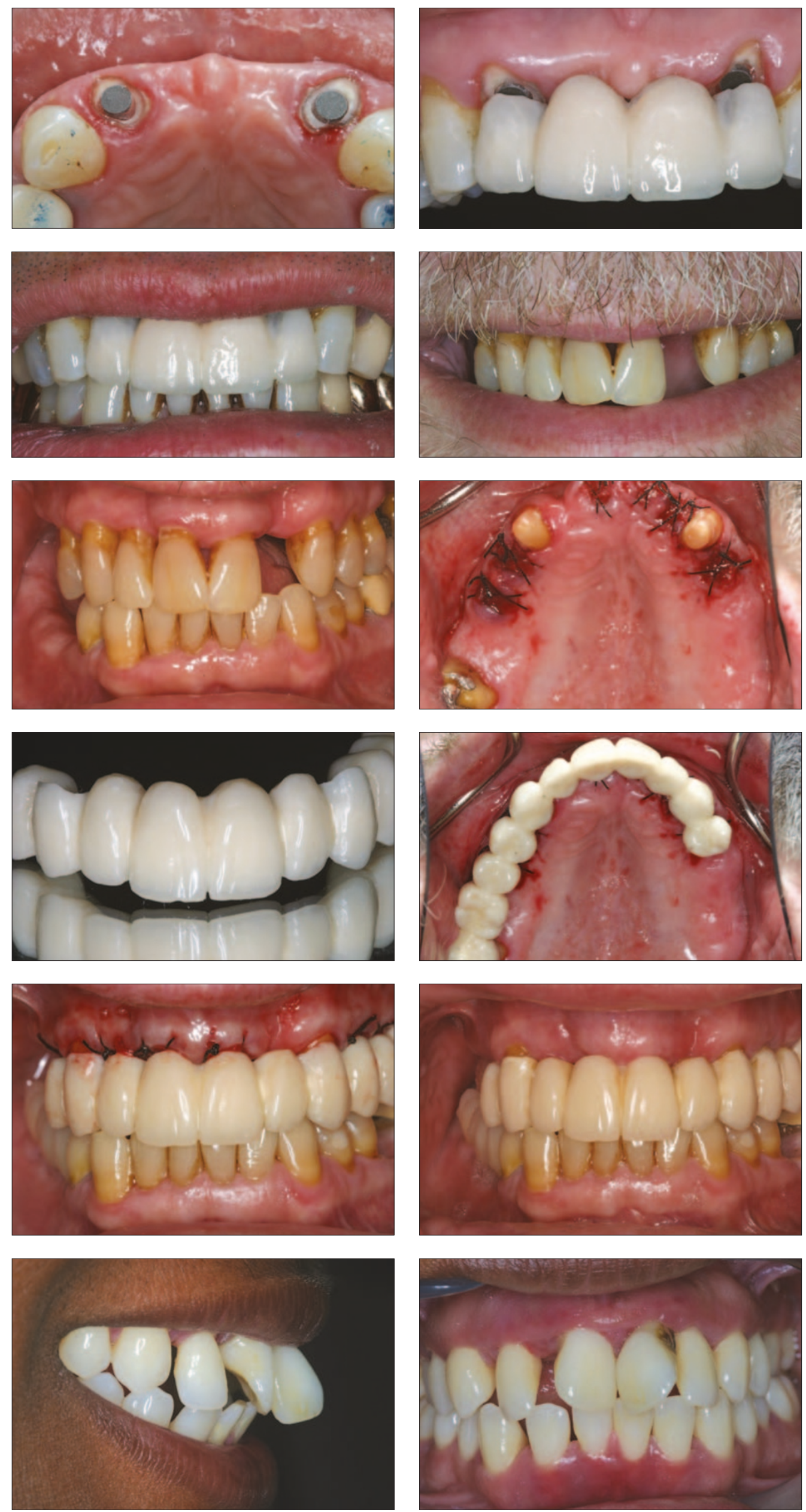
PRACTICE
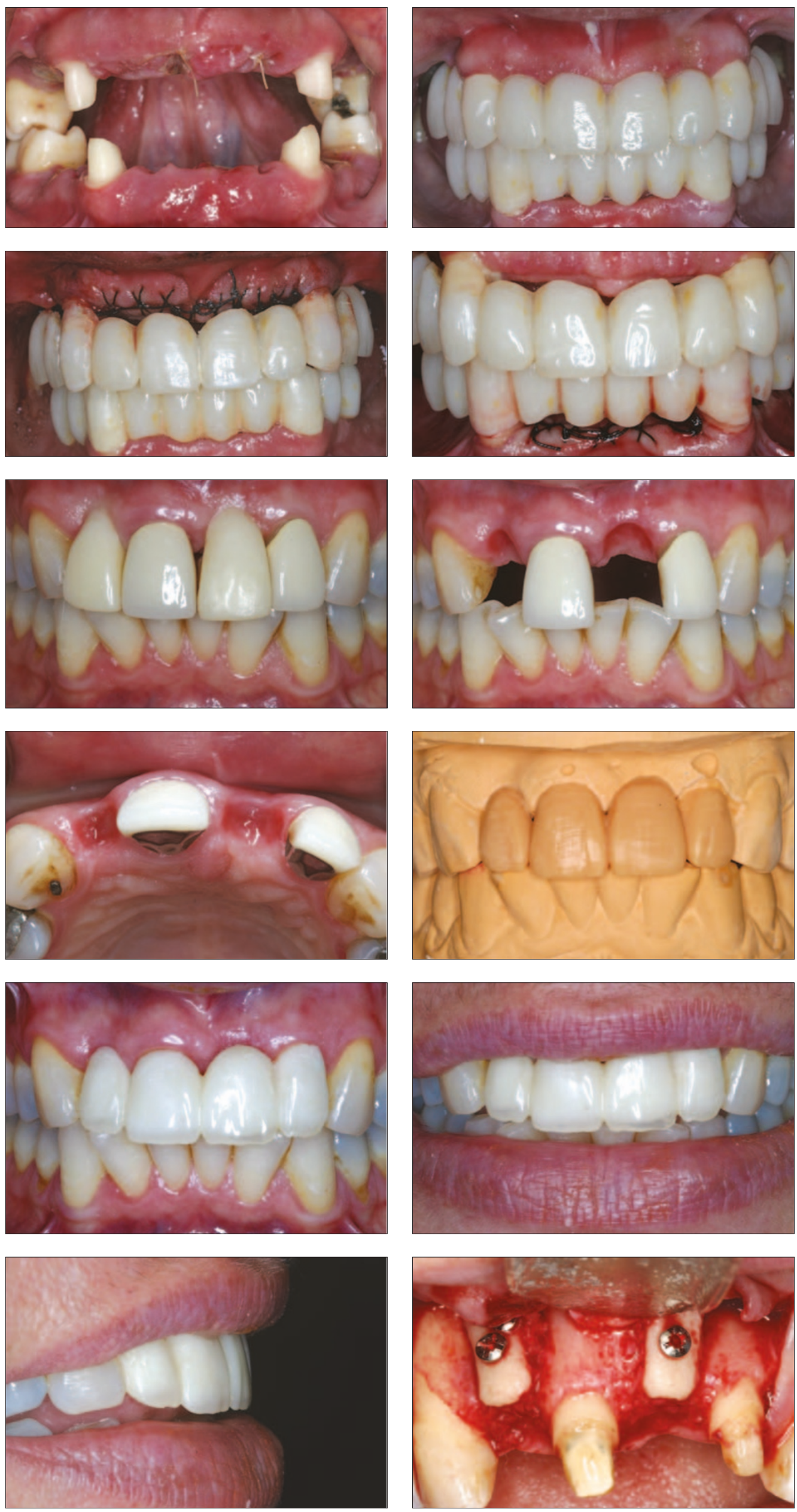

Fig. 25 (left) Incisors and premolars extracted, canines prepared to retain a provisional restoration. The molars have been retained temporarily as a reference for vertical dimension while provisional restorations are delivered.

Fig. 26 (right) First and second molars have been extracted, third molars have been retained as reference point. Provisional restoration delivered on four canine teeth.

\section{Fig. 27 (left) Facial view on day} of maxillary implant placement, provisional has been relined over the implant sites.

Fig. 28 (right) Final view on day of mandibular implant placement, provisionals have been relieved over the implant site.

Fig. 29 (left) Facial view showing interim partial denture replacing teeth 7 and 9. Patient requested implant supported restorations and was dissatisfied with the proportions of the teeth.

Fig. 30 (right) Facial view showing discrepancy in tooth length.

Fig. 31 (left) Occlusal view showing facio-lingual collapse. Patient was treatment planned to receive autogenous bone grafts to optimise the implant site.

Fig. 32 (right) Diagnostic wax up from teeth 7-10 illustrating anticipated contours of final restorations.

Fig. 33 (left) Provisional restoration following removal of crowns on teeth 8 and 10 . This provisional restoration can also function as a radiographic guide.

Fig. 34 (right) Facial view of provisional restorations in situ showing improved contours and proportions.

Fig. 35 (left) Lateral view of provisional restorations in situ demonstrating improved emergence profile.

Fig. 36 (right) Autogenous bone grafts placed in sites 7 and 9 . 
Fig. 37 Provisional restoration placed back in situ, the provisional is adjusted so that all pressure is kept off of the graft site.

Fig. 38 Shell provisional to be relined on transitional implants.

Fig. 39 Occlusal view, shell provisional has acrylic extension which will be indexed on the palate. Provisional is linked with wire to maintain form during reline.

Fig. 40 Relined provisional intraorally, acceptable aesthetics achieved.

Fig. 41 Patient's smile following surgery.

Fig. 42 A complication of immediate loading is fracture of the cantilever portion of the prosthesis. Cantilevers should be minimised.

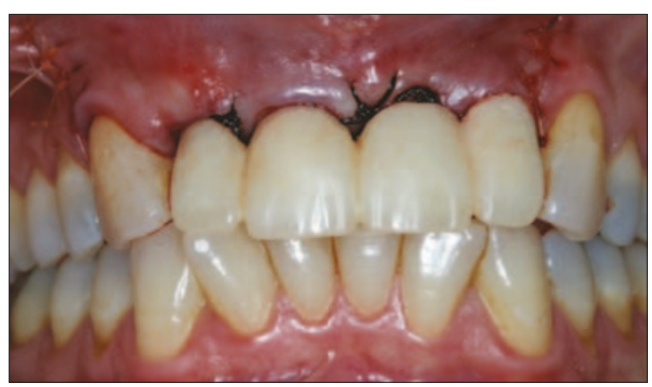

fixed partial denture. Abutments with a poor prognosis are often used as these abutments are eventually being extracted when the implants have integrated. These abutment teeth only need to survive till implants integrate.

Strategic abutments are commonly used in full arch situations when the patient's existing dentition is failing due to periodontal disease. Once a decision has been made to move toward an implant supported reconstruction, all teeth are planned for eventual extraction. Implant sites are selected first, those teeth occupying the implant sites are removed and the remaining teeth are used to support a provisional restoration. The alternative to this would be to edentulate the patient and deliver an immediate denture. The psychological and physiological changes associated with a complete denture can be dramatic. If onlay grafting is required for these types of patients, the pressure exerted by the removable prosthesis can accelerate resorption of the graft and negate the surgeon's initial efforts. Maintaining a few teeth to retain a fixed transitional prosthesis provides both physiological and psychological advantages for the patient, one of the most important being the psychological security of a fixed prosthesis in social settings. (Figs 16-22).

When integration of the implants is confirmed, the strategic abutments are extracted and the reconstruction with implants as the source of support can continue. Advantages of this approach include maintaining the patient in a fixed prosthesis and protecting surgical and implant sites during the healing phase. Disadvantages include increased cost for the patient and the possible adverse sequelae to implant integration should retained abutments become infected during the integration.

The patient in Figure 23 had a periodontally failing dentition and was treatment planned for removal of all teeth and an implant supported reconstruction. The patient was a university age student and when told of this she was devastated; the thought of a complete denture was unbearable and the opportunity to transition to the implant supported option without the use of a complete denture significantly softened the blow and enhanced her acceptance of the treatment. The extractions were carried out in two phases. The canine teeth were prepared to receive a 10-unit fixed transitional prosthesis in each arch. This prosthesis was delivered on the day of extraction of the anterior teeth and premolars. A balanced occlusion was provided. ${ }^{8}$ The molar teeth were maintained to preserve vertical dimension and to provide a point of reference with which to evaluate the occlusion. Once the patient was comfortable with the prosthesis, the first and second molars were extracted. The third molars were maintained till the end of therapy to aid supporting the forces of occlusion and to help stabilise the surgical guides. This allowed the patient to function with a fixed prosthesis during the healing phase and allowed the clinician to protect the implant 
surgical site during the submerged healing phase (Figs 24-28).

In the aesthetic zone a fixed transitional prosthesis also provides benefits. The patient in Figure 29 presented requesting replacement of her removable partial denture replacing teeth 7 (11) and 9 (21) with implant restorations. On clinical and radiographic examination there was inadequate buccolingual width for placement of implants in ideal positions (Figs 30-31). Autogenous bone grafting of sites 7 (11) and 9 (21) was required prior to placement of implants. A diagnostic wax up was completed (Fig. 32) and provisional fixed partial denture fabricated from teeth 7-10 (Figs 33-35). Autogenous bone grafts were completed to augment bone width (Fig. 36-37). The provisional restoration served to maintain aesthetics and relieve the surgical site from any untoward pressure in the healing phase.

\section{Transitional implants}

Immediate loading concepts have been well established. These techniques have been well documented for implant retained mandibular overdentures and for mandibular implant supported complete dentures. ${ }^{9-14}$

Prior to the advent of immediate loading concepts, transitional implants were developed to enable undisturbed healing and fulfil the patient demand for uninterrupted immediate function and aesthetics. The primary function of transitional implants is to absorb masticatory stress during the healing phase, ensuring stress-free maturation of the bone around the submerged implants and allowing them to heal uneventfully. ${ }^{15,16}$ Most importantly patients immediately experience the benefits of implant dentistry and come out of the surgical phase with a fixed prosthesis.

The scope of transitional implants can be expanded into diverse applications such as undisturbed healing of bone grafts and provisionalisation of fully and partially edentulous patients. They are particularly useful in situations when bone quality is not adequate for immediate loading of the definitive implants but the patient requests a fixed transitional prosthesis. For a full arch prosthesis, a sufficient number of transitional implants should be placed to allow survival of the prosthesis should one or two implants fail.

Transitional implants are an effective way to generate aesthetic transitional appliances. For the clinician they allow evaluation of aesthetics, phonetics and function in the interim phase. Patients can return to their daily activities with a fixed restoration and avoid social embarrassment (Figs 38-41).

Despite all the advantages of transitional implants, they should still be used with caution. On occasion the volume of bone used for their placement may be of strategic value during treatment and risks being destroyed by fibrous tissue formation or bone resorption when loaded immediately. Also, if a definitive implant

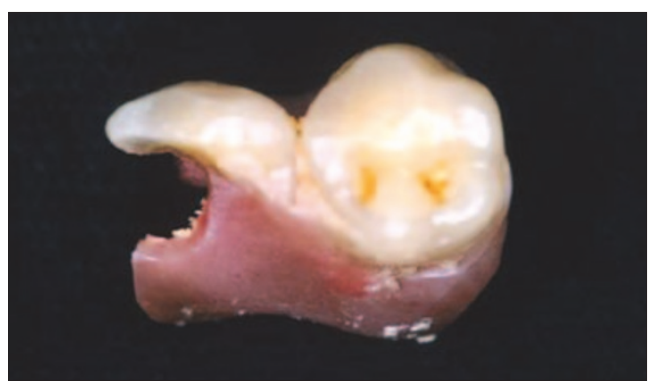

Fig. 43 Cantilever cannot be reattached or the prosthesis removed, the fractured portion must be smoothed in situ.

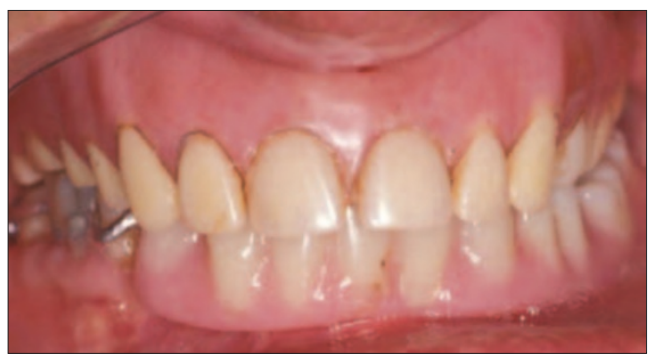

Fig. 44 Pre-operative situation illustrating inadequate maxillary complete and mandibular partial dentures.

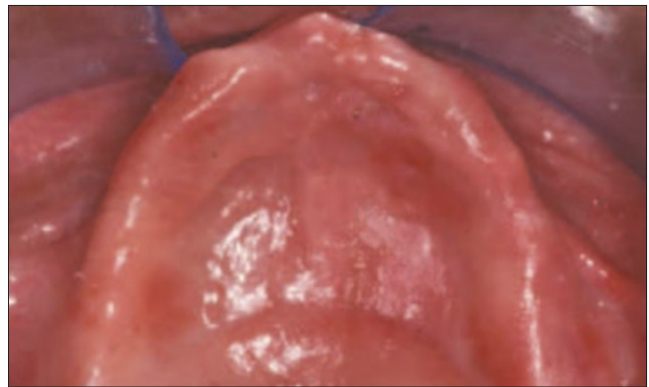

Fig. 45 Occlusal view of palate showing trauma to palate from existing denture. Denture bearing area has also been reduced.

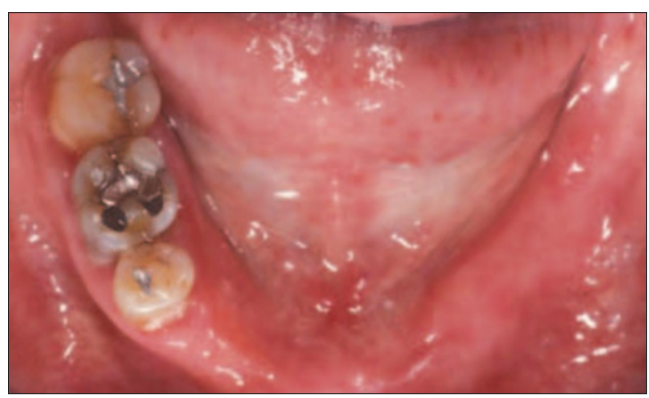

Fig. 46 Occlusal view illustrating edentulous space and existing teeth.

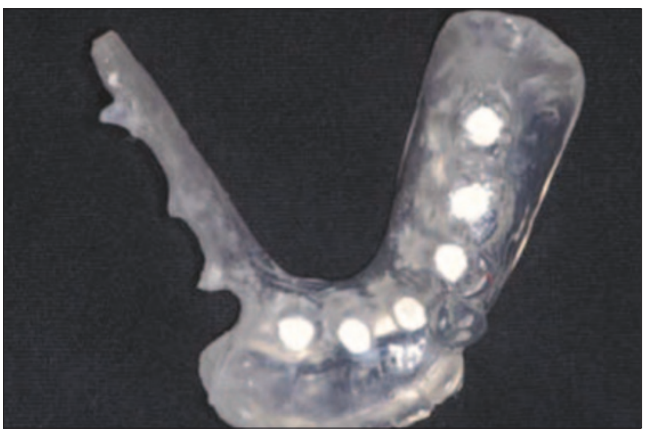

Fig. 47 Radiographic guide.

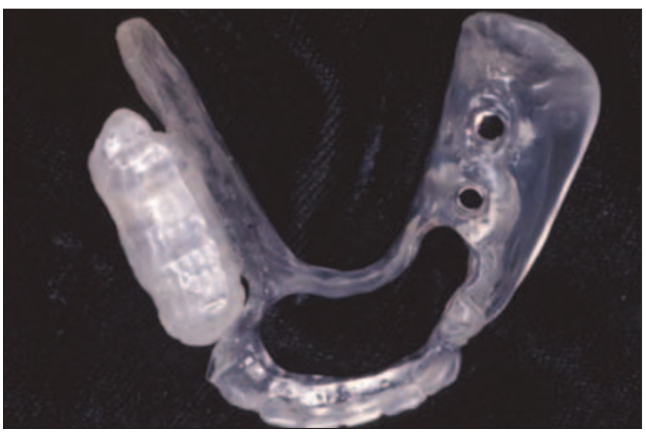

Fig. 48 Surgical guide is fabricated to gain stability from existing teeth. These teeth will be extracted following implant placement. 
Fig. 49 (left) Initial incisions.

Fig. 50 (right) Implants placed on left hand side of patients mandible. Surgical guide gains stability from existing teeth to ensure accurate implant placement.

Fig. 51 (left) Teeth have been extracted and remaining implants placed.

Fig. 52 (right) Temporary cylinders have been attached to transmucosal abutments.

Fig. 53 Mandibular denture is achieved to be free of temporary cylinders.
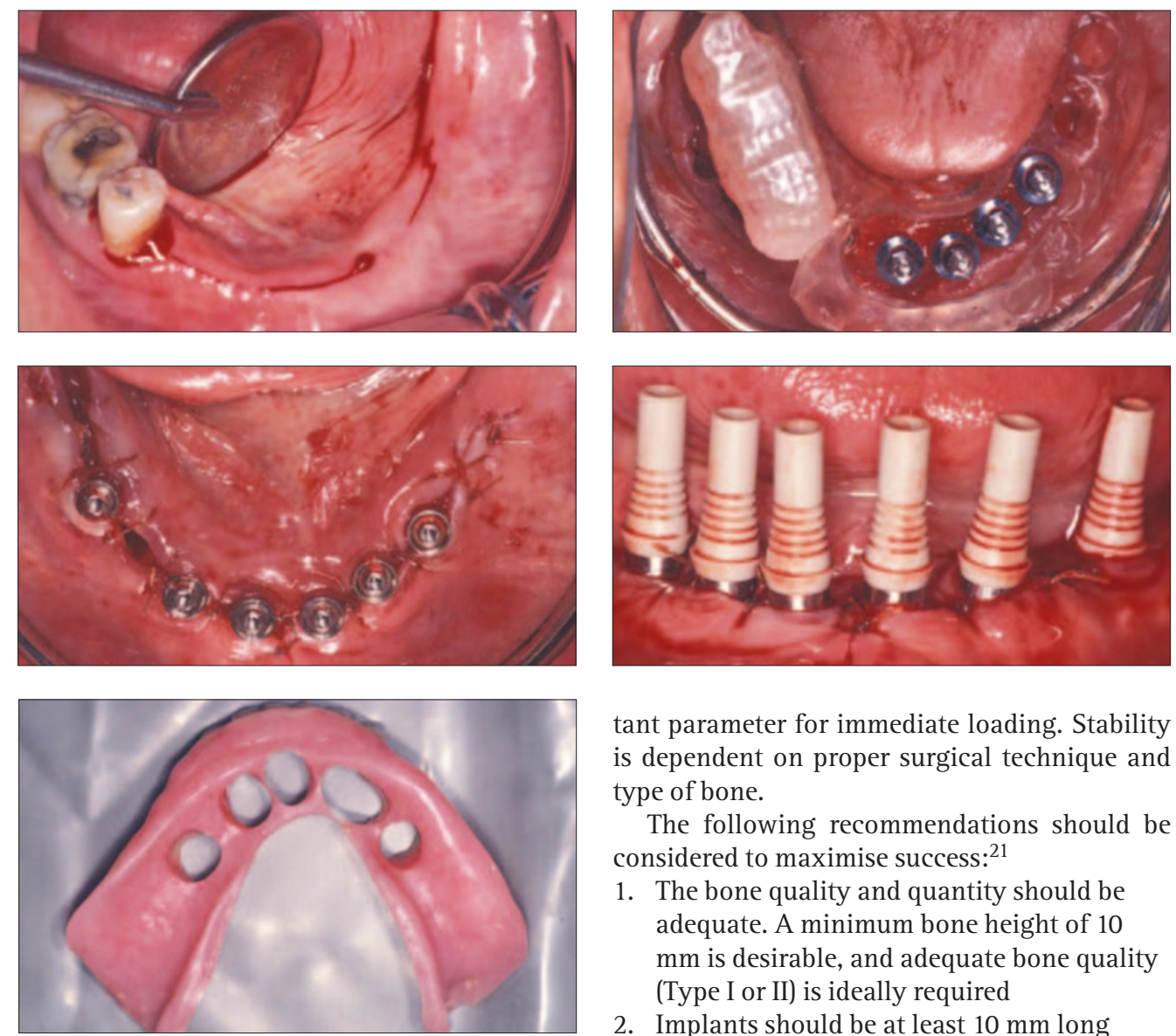

tant parameter for immediate loading. Stability is dependent on proper surgical technique and type of bone.

The following recommendations should be considered to maximise success: ${ }^{21}$

1. The bone quality and quantity should be adequate. A minimum bone height of 10 $\mathrm{mm}$ is desirable, and adequate bone quality (Type I or II) is ideally required

2. Implants should be at least $10 \mathrm{~mm}$ long

3. There should be an adequate number and distribution of implants to provide cross arch stabilisation

fails, the alternate site has already been used and is unavailable.

\section{Immediate loading}

The predictability of dental implants using the traditional Branemark protocol has been well documented. Since its inception, this protocol has been progressively challenged to decrease treatment time, minimise the number of surgical procedures, and maximise aesthetic outcomes. Practitioners should be aware that the Branemark protocol was empirical and based on clinical observation; it was a protocol that insured integration of implants but each and every step may not be necessary. ${ }^{18}$ Treatment of fully edentulous patients with dental implants highlighted many complications during the osseointegration phase. Complications involving complete dentures worn during the period of osseointegration included loose dentures, fractured prosthesis, sore spots and periodic provisional relines. Today, in specific clinical situations, implants may be placed and immediately loaded with provisional restorations. Immediate loading in the edentulous mandible has been well documented. ${ }^{12-14}$ There are also case reports showing that immediate loading of the edentulous maxilla is feasible if bone quality is suitable. ${ }^{19,20}$

Immediate loading is a very effective way of transitioning patients from teeth to a complete full arch implant supported restoration. Based on guidelines from previous studies, ${ }^{12-14}$ primary stability appears to be the most impor-
4. Good initial stability of the implant with minimum insertion torques of $35-50 \mathrm{~cm}^{22}$

5. Passive fit of provisional restoration

6. Sufficient interocclusal space should be present to allow fabrication of a provisional restoration with adequate rigidity

7. Even occlusal contacts

8. Cantilevers should be avoided or minimised to one premolar (Figs 42-43)

9. Removal of the provisional restoration should be avoided during the osseointegration period ${ }^{14}$

10. Patients with parafunctional habits may not be ideal candidates.

Advantages of immediate loading are obvious for the practitioner. The practitioner benefits from a reduced number of post-operative visits and reduced maintenance of the provisional restoration ${ }^{4}$ (Figs 44-58).

Risks of immediate loading are perceived to be during the first week following the insertion of implants. The bone interface is actually stronger on the day of implant placement compared with three months later - this is location dependent. ${ }^{23}$ The risk of immediate loading is implant failure and implant failure can cause the use of a removable prosthesis. If the remaining distribution of implants will not allow proper stabilisation of the provisional restoration, an unstable restoration would jeopardise the remaining implants. Failure also brings with it 

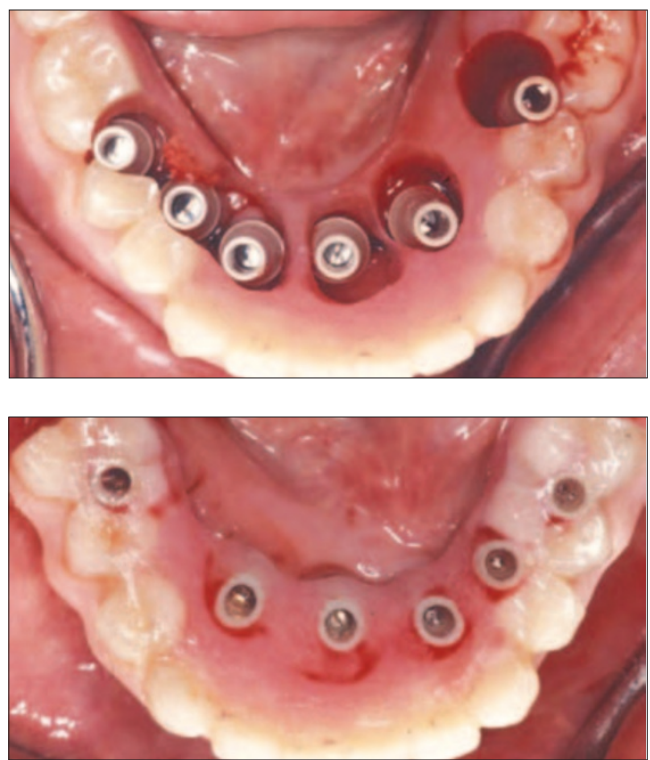

additional appointments and greater chair time for the clinician which translates to increased costs. Patients must be made aware of the risks of failure prior to embarking upon this path: immediate loading is a risk that both patient and clinician must share.

One benefit of immediate loading is function - of late the benefit to aesthetics has come to the fore. Clinicians have recognised that preserving the hard and soft tissue components that exist around natural teeth while transitioning to implants is easier and more predictable than losing these elements with an extraction then trying to re-build them. Techniques which involve immediate extraction, immediate placement and immediate loading have also been advocated. The advantages of immediate restoration are obvious; however, the application of immediate or early load may pose an increased risk of implant failure in single-tooth situations. The parameters for achieving and maintaining equal success rates of osseointegration for single teeth are not fully known.

Sufficient data are available to support the concept that immediately restored and loaded implants in single tooth situations in the aesthetic zone can achieve integration using many implant systems and protocols. ${ }^{24-26}$ Many authors have concluded that aesthetic results in immediately loaded sites are superior to that achieved with a staged approach because of gingival architecture preservation.

Most studies and case reports are carried out by highly trained clinicians working under strict protocols. These protocols respect certain parameters. Factors that have been highlighted to improve the success rate include absence of parafunctional habits, use of a roughened implant surface, use of a threaded implant and most importantly primary stability. Removing occlusal contacts and enforcing a soft diet have also been proposed.

The risk of immediate load in a single tooth situation should be obvious to the clinician. The risk of failure is a costly one which has both

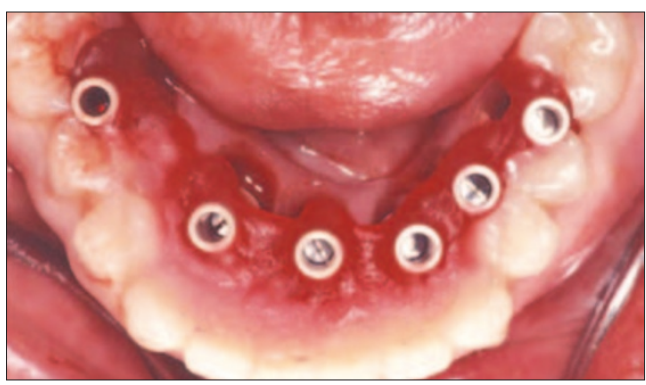

Fig. 54 (left) Try in of mandibular denture over temporary cylinders.

Fig. 55 (right) Mandibular denture attached to temporary cylinders using duralay resin.

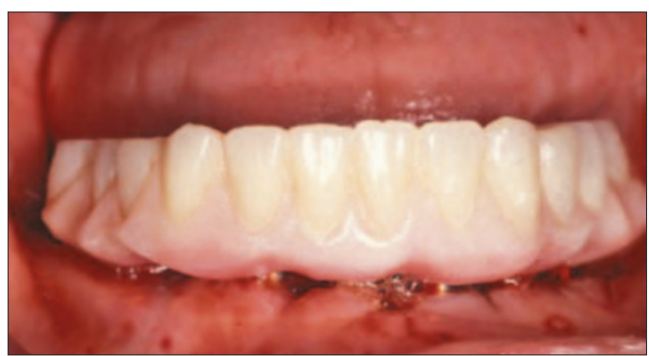

Fig. 56 (left) Denture taken to lab and remainder of space filled in with self cured denture resin.

Fig. 57 (right) Facial view of screw retained immediate load prosthesis. Prosthesis has been adjusted to allow the patient to maintain hygiene.

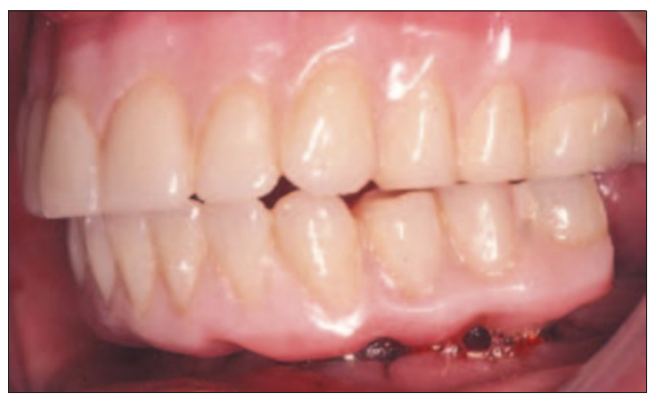

Fig. 58 Lateral view of transitional maxillary denture and mandibular immediate load prosthesis. Occlusion has been adjusted to ensure bilateral simultaneous contact. biological and financial repercussions. Many studies and case reports do not address the consequences of failure and how the patient is managed.

It is prudent to ask:

1. Is immediate loading in the aesthetic zone really worth the risk?

2. Does delaying delivery of the restoration disadvantage the patient?

Immediate loading in the aesthetic zone is becoming ever more accepted. The clinician has a chance of success with adequate treatment planning, incorporating as much clinical data as possible and understanding the limitations imposed. Patient selection and risk/benefit analysis for each patient will lead to more predictable and desirable outcomes.

\section{SUMMARY}

An axiom of traditional prosthodontics is to provide a fixed prosthesis whenever possible. When patients are going through lengthy and costly implant therapy their expectations for both function and aesthetics are high. This expectation stands for both the provisional and definitive restoration. Patients prefer to avoid wearing removable restorations in the transitional phase. The advantages of this have been previously outlined. There are many options to transition a patient from tooth supported to implant supported restorations using a fixed transitional prosthesis. The increased costs are 
offset by the practical and psychological convenience of a fixed prosthesis. Patients realise the benefits of implant therapy immediately and are more likely to be accepting of treatment plans involving extensive implant restorations.

The authors would like to thank Dr Eva Berroeta for the slides in Figures 42 and 43.

1. Branemark P-I, Zarb G A, Albrektsson T. Tissue integrated prostheses: osseointegration in clinical dentistry. Carol Stream IL: Quintessence, 1985

2. Adell R, Lekholm U, Rockler B, Branemark PI. A 15 year study of osseointegrated implants in the treatment of the edentulous jaw. Int J Oral Surg 1981; 10: 387-416.

3. Sullivan R M. Perspectives on aesthetics in implant dentistry. Compend Contin Educ Dent 2001: 22: 685-692.

4. Chee W W L, Jivraj S. Efficiency of immediately loaded mandibular full arch restorations. Clin Implant Dent Relat Res 2003; 5: 52-56.

5. Rochette A L. Attachment of a splint to enamel of lower anterior teeth. J Prosthet Dent 1973; 30: 418-423.

6. Livaditis G J. Thompson V P. Etched castings. An improved retentive mechanism for resin bonded retainers. J Prosthet Dent 1982; 47: 52-58.

7. Creugers N H J, Van't Hof M A. An analysis of clinical studies on resin bonded bridges. J Dent Res 1993 : 72: 1244-1248.

8. Lindhe J, Nyman S. Long term maintenance of patients treated for advanced periodontal disease. J Clin Periodontol 1984; 11(8): 504-514

9. Leklove M D, Beals R P. Immediate loading of cylinder implants with overdentures in the mandibular symphysis: the titanium plasma sprayed screw technique. J Oral Implantol 1990; 16: 265-271.

10. Hruska A B, Borelli P. Intra-oral welding of implants for an immediate loading with overdentures. J Oral Implantol 1993: 14:34-38.

11. Chiapasco M, Gatti C, Rossi E, Haefliger W, Markwalder TH. Implant retained mandibular overdentures with immediate loading. A retrospective multicenter study on 226 consecutive cases. Clin Oral Implants Res 1997; 8: 48-57.

12. Schnitman PA, Wohrle PS, Rubenstein J S. Immediate fixed interim prostheses supported by two stage threaded implants: methodology and results. J Oral Implanto/ 1990; 16: $96-105$.
13. Schnitman PA, Wohrle PS, Rubenstein J E, DaSilva $J$ D, Wang N H. Ten year results for Branemark implants immediately loaded with fixed prosthesis at implant placement. Int J Oral Maxillofac Implants 1997; 12: 495-503.

14. Tarnow D P, Emtiaz S, Classi A. Immediate loading of threaded implants at stage 1 surgery in edentulous arches: ten consecutive case reports with 1-5 year data. Int J Oral Maxillofac Implants 1997; 12:310-324.

15. Froum S, Emtiaz S, Bloom M J, Scolnick J, Tarnow D P. The use of transitional implants for immediate fixed temporary prostheses in cases of implant restorations. Pract Periodontics Aesthet Dent 1998; 10: 737-746.

16. Bichacho N, Landsberg C J, Rohrer M, Davidovich Y. Immediate fixed transitional restoration in implant therapy. Pract Periodontics Aesthet Dent 1999; 11: 45-51.

17. Misch C E. Dental implant prosthetics. pp 182. USA: Mosby, 2005.

18. Szmukler-Moncler S, Piatelli A, Favero G A, Dubruille J H. Considerations preliminary to the application of early and immediate loading protocols in dental implantology. Clin Oral Implants Res 2000; 11 : 12-25.

19. Grunder U. Immediate functional loading of immediate implants in edentulous arches: two-year results. Int J Periodontics Restorative Dent 2001; 21: 545-551.

20. Jaffin R A, Kumar A, Berman C L. Immediate loading of dental implants in the completely edentulous maxilla: a clinical report. Int J Oral Maxillofac Imp 2004; 19: 721-730.

21. Derbabian K, Simonian K. Immediate loading of dental implants: overview and rationale. J Calif Dent Assoc 2005; 33: 337-341.

22. Brunski J B. Avoid pitfalls of overloading and micromotion of intraosseous implants. Dent Implantol Update 1993; 4: 77-81.

23. Strid KG. Radiographic results. In Branemark PI, Zarb G A, Albrektsson T (eds). Tissue integrated prosthesis. Chicago: Quintessence, 1985.

24. Malo P, Rangert B, Dvarsater L. Immediate function of Branemark implants in the aesthetic zone: a retrospective clinical study with 6 months to 4 years of follow-up. Clin Implant Dent Relat Res 2000; 2: 138-146.

25. Rocci A, Martignoni M, Gottlow J, Rangert B. Immediate function of single and partial reconstructions in the maxilla using Mk IV fixtures. A retrospective analysis. App/ Osseointegration Res 2001; 2: 22-43.

26. Wohrle PS. Single-tooth replacement in the aesthetic zone with immediate provisionalization: fourteen consecutive case reports. Pract Periodontics Aesthet Dent 1998; 10: $1107-1114$ 